\title{
Duration of previous treatment as a prognostic factor in metastatic colorectal cancer treated with trifluridine/tipiracil
}

\author{
ELINA SKUJA $^{1,2}$, AIJA GERINA-BERZINA ${ }^{1,3}$, ALINTA HEGMANE $^{3,4}$, \\ ZANETE ZVIRBULE ${ }^{4}$, EVA VECVAGARE ${ }^{4}$ and GUNTA PURKALNE ${ }^{1,2}$ \\ ${ }^{1}$ Clinic of Oncology, Pauls Stradins Clinical University Hospital, LV-1002 Riga; ${ }^{2}$ Oncology Department, \\ Riga Stradins University, LV-1007 Riga; ${ }^{3}$ Oncology Department University of Latvia, LV-1050 Riga; \\ ${ }^{4}$ Oncology Centre, Riga East University Hospital, LV-1079 Riga, Latvia
}

Received January 8, 2018; Accepted March 9, 2018

DOI: $10.3892 / \operatorname{mco} .2018 .1600$

\begin{abstract}
We herein describe the findings from the trifluridine/tipiracil (TAS-102) Compassionate Use program in Latvia, set up prior to marketing authorization for the management of pretreated patients with metastatic colorectal cancer (mCRC). The efficacy and safety of TAS-102 in patients with refractory $\mathrm{mCRC}$ were evaluated in the phase III trial RECOURSE. A previous report confirmed neutropenia and duration of previous treatment for $\mathrm{mCRC}$ as prognostic factors in TAS-102 users. The aim of the present study was to analyze possible prognostic factors, such as neutropenia, in TAS-102 responders. A retrospective analysis of 14 patients who received TAS-102 chemotherapy in two institutions in Latvia (Clinic of Oncology of Pauls Stradins Clinical University Hospital and Oncology Centre of Riga East University Hospital) was performed. Grade 3-4 neutropenia was observed in $28 \%$ of the patients. In patients with grade 3-4 neutropenia the median progression-free survival (mPFS) was 7 months, whereas in those without neutropenia the mPFS was 5 months [hazard ratio $(\mathrm{HR})=0.24, \mathrm{P}=0.033]$. In $64 \%$ of the patients, the duration of previous treatment was $>18$ months. In patients treated for $>18$ months from the start of first-line mCRC treatment the mPFS was 7 months, whereas in those treated for $\leq 18$ months from the start of first-line mCRC treatment the mPFS was 5 months $(\mathrm{HR}=0.15, \mathrm{P}=0.029)$. Therefore, a longer time from mCRC diagnosis until disease progression may be used to select chemotherapy-refractory mCRC patients for TAS-102 treatment. Furthermore, severe neutropenia may be considered as a surrogate marker for predicting TAS-102 treatment outcomes.
\end{abstract}

Correspondence to: Dr Elina Skuja, Clinic of Oncology, Pauls Stradins Clinical University Hospital, 13 Pilsonu Street, LV-1002 Riga, Latvia

E-mail: elinaskuja@inbox.lv

Key words: trifluridine/tipiracil, TAS-102, colorectal cancer, refractory

\section{Introduction}

Metastatic colorectal cancer (mCRC) is one of the leading types of cancer in developed countries. Despite treatment and diagnostic advances, the mortality rates of $\mathrm{mCRC}$ remain high, with a patient survival of 24-30 months. In Latvia, 1,000 patients with colon and rectum cancer are diagnosed annually, $25 \%$ of whom are metastatic at presentation (1).

Cytotoxic agents, including a fluoropyrimidine, irinotecan and oxaliplatin, and antibodies, such as bevacizumab (an antivascular endothelial growth factor monoclonal antibody) and cetuximab and panitumumab (anti-epidermal growth factor receptor monoclonal antibodies) may significantly improve the survival of patients with unresectable mCRC. However, chemotherapy-refractory $\mathrm{mCRC}$ patients represent a major challenge for medical oncologists in terms of selection of further treatment.

Trifluridine/tipiracil (FTD/TPI), also referred to as TAS-102, is a combination of an antineoplastic thymidinebased nucleoside analog, trifluridine, and the thymidine phosphorylase inhibitor tipiracil hydrochloride at a molar ratio of 1:0.5. The efficacy and safety of FTD/TPI in patients with mCRC who are refractory to standard therapies were evaluated in the RECOURSE trial (2).

\section{Patients and methods}

Study group. A total of $14 \mathrm{mCRC}$ patients who received FTD/TPI chemotherapy in two institutions in Latvia (Clinic of Oncology of Pauls Stradins Clinical University Hospital and Oncology Centre of Riga East University Hospital) were analyzed. The study was performed with the Lonsurf Compassionate Use program (Expanded Access program) and written informed consent was obtained from each patient who participated in the study. The inclusion criteria were as follows: Metastatic cancer of the colorectum, neutrophil count $>1,500 / \mathrm{mm}^{3}$, platelet count $>75.000 / \mathrm{mm}^{3}$, hemoglobin level $>9.0 \mathrm{~g} / \mathrm{dl}$, at least 2 previous chemotherapy lines, refractory to or intolerant of fluoropyrimidines, oxaliplatin and irinotecan. The patient inclusion period was only 1 month (April 2016), and a maximum of 15 patients were allowed to participate in this program in Latvia. Data on clinical follow-up were 
obtained until June 2017. The patients underwent 1-13 months of follow-up.

Treatment. FTD/TPI (with each dose consisting of $35 \mathrm{mg} / \mathrm{m}^{2}$ ) was administered orally twice daily, for 5 days a week, with 2 days of rest, for 2 weeks, followed by a 14-day rest period, thus completing one cycle. The regimen was repeated every 4 weeks. The dose was recommended as standard in all sites participating in the Lonsurf Compassionate Use program (2). Treatment was discontinued upon clinically or radiologically confirmed disease progression.

Statistical analysis. Progression-free survival (PFS) was calculated from the start of FTD/TPI until clinical or radiological progression, and overall survival (OS) was calculated from the start of FTD/TPI until death from any cause or censoring at the last follow-up. The median OS and PFS (mOS and mPFS, respectively) were estimated using the Kaplan-Meier method. The log-rank test was used to calculate any significant differences between the subgroups (patients with vs. without grade 3-4 neutropenia, and duration of previous treatment $\leq$ vs. $>18$ months) by univariate analysis. Significance levels were set at $\mathrm{P}<0.05$. All statistical analyses were performed by MedCalc software, version 16.4.8 (MedCalc Software, Ostend, Belgium).

\section{Results}

Patients. Of the 15 patients, 14 received FTD/TPI treatment in the Lonsurf Compassionate Use program in Latvia between April 2016 and January 2017 in the two participating institutions (1 patient was excluded from the program due to rapid cancer progression). The clinical characteristics of the participants are listed in Table I. A total of 8 patients (57.2\%) had primary metastatic cancer (stage IV), and 6 patients developed metastases after treatment of the primary cancer. All the patients had received 2-4 previous lines of chemotherapy and progressed.

Treatment and adverse events. The staring dose of FTD/TPI was $35 \mathrm{mg} / \mathrm{m}^{2}$, and the duration of treatment was $1-9$ cycles (median, 5.8 cycles).

One patient had a delay of 1 week due to grade 3 neutropenia; none of the patients required dose reduction, and all patients eventually discontinued FTD/TPI due to disease progression. All treatment-related adverse events are listed in Table II.

Survival data. At the median follow-up time of 7.1 months (range, 1-14 months), the mPFS was 5 months [95\% confidence interval (CI): 4.09-5.90], and the mOS was 7 months (95\% CI: 5.95-8.04). The 6-month PFS was $35.7 \%$ and the 6-month OS was $57.1 \%$. All 14 patients progressed on FTD/TPI treatment and 9 deaths were reported.

Increased PFS and OS were reported in patients with grade 3-4 neutropenia: The mPFS was 7 months in patients with neutropenia vs. 5 months in those without neutropenic events [hazard ratio $(\mathrm{HR})=0.24,95 \% \mathrm{CI}$ : $0.07-0.89 ; \mathrm{P}=0.033$ ]; the mOS was 7 months in patients without neutropenia, whereas in the neutropenic group mOS was not met $(\mathrm{HR}=0.25,95 \% \mathrm{CI}$ : 0.06-1.14, P=0.075; Fig. 1).

Furthermore, increased PFS and OS were observed in patients with a time of $>18$ months from the start of
Table I. Patient clinical characteristics $(n=14)$.

\begin{tabular}{|c|c|}
\hline Characteristics & No $(\%)$ \\
\hline \multicolumn{2}{|l|}{ Age, years } \\
\hline Median (range) & $65(52-76)$ \\
\hline \multicolumn{2}{|l|}{ Sex } \\
\hline Male & $6(42.8)$ \\
\hline Female & $8(57.2)$ \\
\hline \multicolumn{2}{|l|}{ ECOG PS } \\
\hline 0 & $5(35.7)$ \\
\hline 1 & $9(64.3)$ \\
\hline \multicolumn{2}{|l|}{ Primary site } \\
\hline Colon & $9(64.3)$ \\
\hline Rectum & $5(35.7)$ \\
\hline \multicolumn{2}{|l|}{ KRAS status } \\
\hline Wild-type & $1(7.1)$ \\
\hline Mutant & $4(28.6)$ \\
\hline Unknown & $9(64.3)$ \\
\hline \multicolumn{2}{|l|}{ Metastases } \\
\hline Synchronous - stage IV at diagnosis & $8(57.2)$ \\
\hline Metachronous & $6(42.8)$ \\
\hline Median time to metastases, months (range) & $22(8-36)$ \\
\hline Stage II at diagnosis & 1 \\
\hline Stage III at diagnosis & 5 \\
\hline $\begin{array}{l}\text { Median time from start of first-line } \\
\text { chemotherapy, month (range) }\end{array}$ & $32.2(8-90)$ \\
\hline$>18$ & $9(64.3)$ \\
\hline$\leq 18$ & $5(35.7)$ \\
\hline Median number of prior lines (range) & $2.7(2-4)$ \\
\hline \multicolumn{2}{|l|}{ Prior chemotherapeutic agents } \\
\hline $5-\mathrm{FU}$ & $14(100.0)$ \\
\hline Ftorafur & $3(21.4)$ \\
\hline Capecitabine & $3(21.4)$ \\
\hline Oxaliplatin & $13(92.8)$ \\
\hline Irinotecan & $14(100.0)$ \\
\hline Bevacizumab & $6(42.8)$ \\
\hline Aflibercept & $1(7.1)$ \\
\hline Cetuximab & $1(7.1)$ \\
\hline
\end{tabular}

ECOG, Eastern Cooperative Oncology Group; PS, performance status; KRAS, K-ras proto-oncogene; 5-FU, 5-fluorouracil.

chemotherapy for mCRC, with a mPFS of 7 months vs. 5 months in patients with a shorter ( $\leq 18$ months) previous treatment duration $(\mathrm{HR}=0.15,95 \% \mathrm{CI}$ : 0.03-0.83, $\mathrm{P}=0.029)$; the mOS was not met in patients with a time of $>18$ months, whereas it was 6 months in the $\leq 18$ months patient group (HR=0.23, 95\% CI: 0.05-1.12, $\mathrm{P}=0.069$; Fig. 2).

\section{Discussion}

The aim of the present study was to compare survival and clinical data from the participants in the Lonsurf Compassionate 

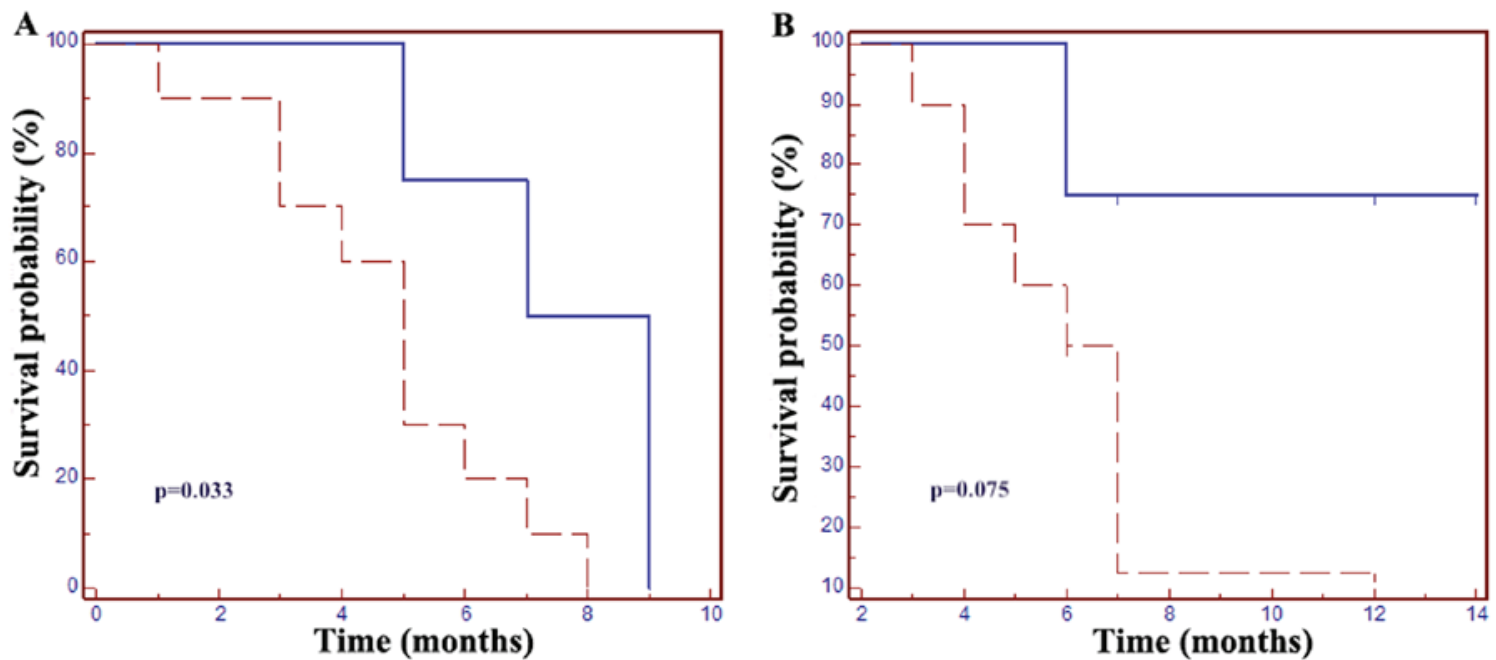

Figure 1. Grade 3-4 neutropenia is a prognostic factor in patients with metastatic colorectal cancer. (A) The mPFS was 5 months in patients without neutropenic events vs. 7 months in the neutropenia group (HR=0.24, 95\% CI: 0.07-0.89; $\mathrm{P}=0.033$. (B) The mOS was 7 months in patients without neutropenia, but in the neutropenic group mOS was not met $(\mathrm{HR}=0.25,95 \% \mathrm{CI}$ : 0.06-1.14, $\mathrm{P}=0.075)$. Dotted line, grade $\leq 2$ neutropenia; solid line, grade $\geq 3$ neutropenia. $\mathrm{mPFS}$, median progression-free survival; mOS, median overall survival; HR, hazard ratio; CI, confidence interval.
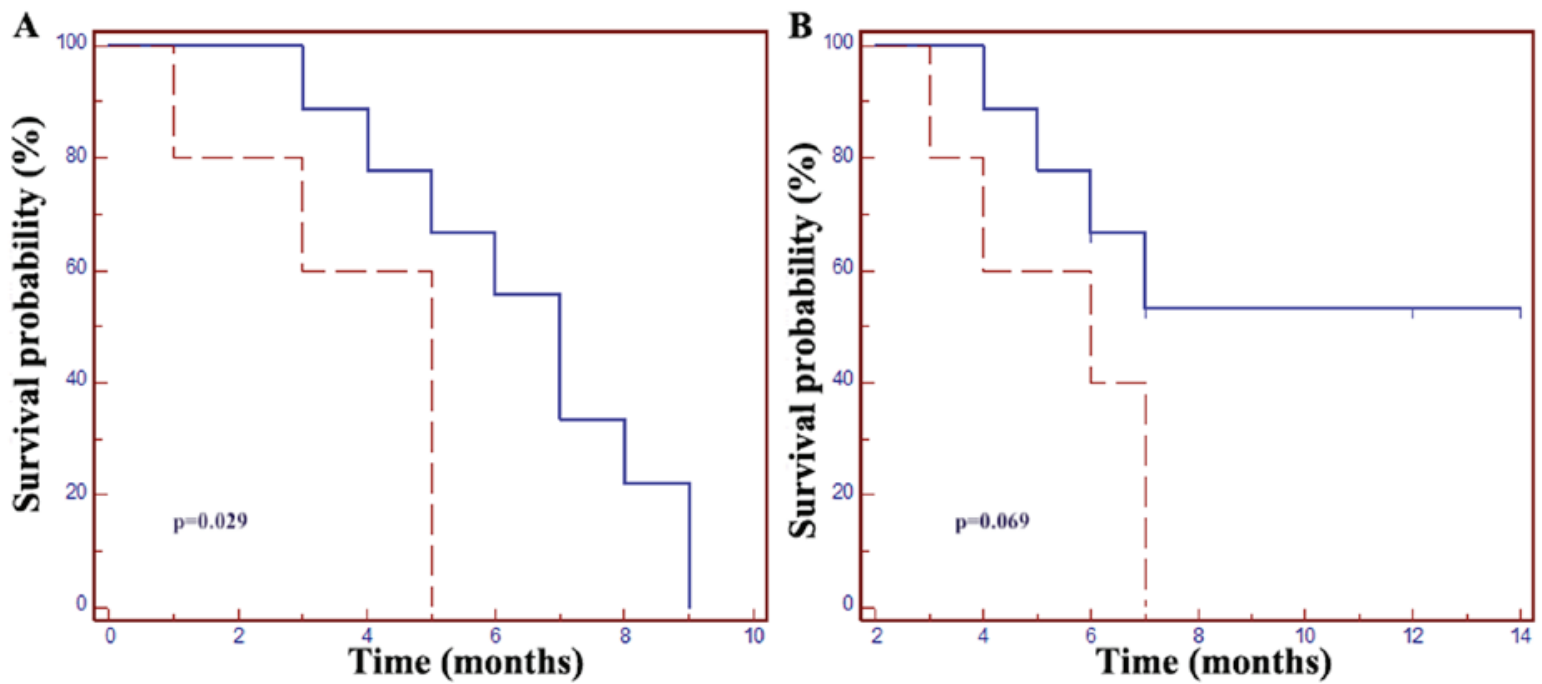

Figure 2. (A) The mPFS in patients with a time of $>18$ months from the start of first-line mCRC treatment was 7 months vs. 5 months in those with a time of $\leq 18$ months (HR=0.15, 95\% CI: 0.03-0.83; $\mathrm{P}=0.029$ ). (B) The mOS in patients with a time of $\leq 18$ months from the start of first-line mCRC treatment was 6 months, but the mOS was not met in patients with a time of $>18$ months ( $\mathrm{HR}=0.23,95 \%$ CI: $0.05-1.12, \mathrm{P}=0.069)$. Dotted line, time $\leq 18$ months from the start of first-line mCRC treatment; solid line, time $>18$ months from the start of first-line mCRC treatment. mCRC, metastatic colorectal cancer; mPFS, median progression-free survival; mOS, median overall survival; HR, hazard ratio; CI, confidence interval.

Table II. Adverse events $(\mathrm{n}=14)$.

\begin{tabular}{lc}
\hline Adverse event & No (\%) \\
\hline Neutropenia, & $4(28.5)$ \\
Diarrhea, any grade & $1(7.1)$ \\
Nausea, any grade & $5(35.7)$ \\
\hline
\end{tabular}

Use program and to report possible prognostic factors for better treatment response. In total, 2,093 patients participated in the Lonsurf Compassionate Use program in 20 countries (3). Previously published data from a phase III trial confirmed the efficacy of FTD/TPI in patients with pretreated mCRC, as treatment with FTD/TPI was associated with a significant improvement in mOS ( 7.1 vs. 5.3 months; $\mathrm{P}<0.0001$ ) and mPFS (2.0 vs. 1.7 months; $\mathrm{P}<0.0001)$ vs. placebo, with a disease control rate of $44 \%$ (2). Similar survival data were observed in the present retrospective study, with a mPFS of 5 months and a mOS of 7 months. The reports from other counties that participated in this Expanded Access program (4-6), revealed a similar trend in safety and efficacy.

In the present study, grade 3-4 neutropenia was the most frequently observed clinically meaningful adverse event, occurring in $28 \%$ of the patients. This adverse event was associated with better treatment outcomes (mPFS of 7 months and increased mOS). Previously reported data 
demonstrated that, in patients with advanced colorectal cancer, FTD/TPI-induced severe neutropenia was associated with superior survival $(2,6-8)$. The reported frequency of grade $3-4$ neutropenia in FTD/TPI users is $31-50 \%$ (2,7-9). The global incidence of grade 3-4 neutropenia is quite low compared to the RECOURSE study, whereas the mPFS is relatively high.

Despite the small number of included patients, it was observed that the time from the start of first-line chemotherapy to the start of FTD/TPI treatment may be a prognostic factor, as the mPFS in patients with a time of $>18$ months from the start of mCRC treatment was 7 months $(\mathrm{HR}=0.15)$, and the HR for cancer-specific mortality was 0.23 (mOS was not met). It may be suggested that patients with a shorter duration of previous treatment lines and rapid progression under previous chemotherapy may benefit less from FTD/TPI treatment. This observation may lead to the hypothesis that more aggressive and chemotherapy-resistant tumors possibly harbor mutations leading to FTD/TPI resistance. Therefore, a variety of previous chemotherapy lines, patient performance status, primary tumor and metastases location may affect the duration of PFS and OS.

There were certain limitations to the present study. Due to the limited number of participants in the Lonsurf Compassionate Use program, the research was conducted on a small size of the mCRC population $(n=14)$. Therefore, to generalize the results for a larger population, the study would require more participants.

In conclusion, severe neutropenia may be considered as a surrogate marker for predicting FTD/TPI treatment outcomes. In addition, patients with a time of $<18$ months from the start of first-line mCRC treatment to the first study drug administration have a poor prognosis. However, further studies are required to confirm these findings.

\section{Acknowledgements}

Not applicable.

\section{Funding}

The article processing charges were funded by the Latvian Association of Medical Oncologists.

\section{Availability of data and materials}

The analysed data sets generated during the study are available from the corresponding author on reasonable request. All authors had full access to all the data in this study and take complete responsibility for the integrity of the data and accuracy of the data analysis.

\section{Authors' contributions}

All named authors meet the International Committee of Medical Journal Editors (ICMJE) criteria for authorship for this manuscript, take responsibility for the integrity of the work as a whole, and have given final approval for the version to be published. ES participated in Lonsurf Compassionate Use program, managed the database, computed results and wrote the paper. All other authors participated in the Lonsurf Compassionate Use program, interpreted the results, commented on the manuscript and approved the final version.

\section{Ethics approval and consent to participate}

This article does not contain any new studies with human or animal subjects performed by any of the authors. The study was performed with the Lonsurf Compassionate Use program (Expanded Access program) approved by the State Agency of Medicines of Latvia. Written informed consent was obtained from each patient who participated in the study.

\section{Consent for publication}

Written informed consent was obtained from each patient who participated in the study.

\section{Competing interests}

The authors declare that they have no competing interests.

\section{References}

1. Latvian cancer registry: https://www.spkc.gov.lv/upload/ Veselibas\%20aprupes\%20statistika/Gadagramata/2015/3_sabiedribas _veseliba_2015_1.pdf. Accessed March 20, 2018.

2. Mayer RJ, Van Cutsem E, Falcone A, Yoshino T, Garcia-Carbonero R, Mizunuma N, Yamazaki K, Shimada Y, Tabernero J, Komatsu Y, et al; RECOURSE Study Group: Randomized trial of TAS-102 for refractory metastatic colorectal cancer. N Engl J Med 372: 1909-1919, 2015.

3. Salvatore L, Niger M, Bellu L, Tamburini E, Garcia-Alfonso P, Amellal N, Delmas AS, Wahba M and Prager G: Compassionate use program for trifluridine/tipiracil (TAS-102) in metastatic colorectal cancer: A real-life overview. Ann Oncol 27 (suppl_6): 512P, 2016

4. Fernández CC, Roces LV, López EL, Ferreras LR, de Segura Iriarte AG and María CR: DI-064 Evaluationof tas-102 as an expanded access in refractory colorectal cancer. Eur J Hosp Pharm Sci Pract 24: A141-A142, 2017.

5. O'Brien C, Callaghan S, Papaxoinis G, Bennett J, Lee CS, Evans RM, Iveson T, Adams R and Mullamitha S: TAS 102 in refractory metastatic colorectal cancer: UK Expanded Access Programme experience. J Clin Oncol 35: e15043, 2017.

6. Kotani D, Pashtoon K, Cecchini M, Shitara K, Ohtsu A, Ramanathan R, Hochster H, Grothey A and Yoshino T: PD-010 Association between chemotherapy-induced neutropenia at 1-month and overall survival in patients receiving TAS-102 for metastatic colorectal cancer. Ann Oncol 27 (suppl 2): ii105, 2016.

7. Hamauchi S, Yamazaki K, Masuishi T, Kito Y, Komori A, Tsushima T, Narita Y, Todaka A, Ishihara M, Yokota T, et al: Neutropenia as a predictive factor in metastatic colorectal cancer treated with TAS-102. Clin Colorectal Cancer 16: 51-57, 2017.

8. Kimura M, Usami E, Iwai M, Teramachi $H$ and Yoshimura T: Severe neutropenia: A prognosticator in patients with advanced/ recurrent colorectal cancerunderoral trifluridine-tipiracil (TAS-102) chemotherapy. Pharmazie 72: 49-52, 2017.

9. Yoshino T, Mizunuma N, Yamazaki K, Nishina T, Komatsu Y, Baba H, Tsuji A, Yamaguchi K, Muro K, Sugimoto N, et al: TAS-102 monotherapy for pretreated metastatic colorectal cancer: A double-blind, randomised, placebo-controlled phase 2 trial. Lancet Oncol 13: 993-1001, 2012. 